\title{
Comparison of spatial domain optimal trade-off maximum average correlation height (OT-MACH) filter with scale invariant feature transform (SIFT) using images with poor contrast and large illumination gradient
}

\author{
A. Gardezi ${ }^{1}$, T. Qureshi , A. Alkandri , R. C. D. Young, P. M. Birch , C. R. Chatwin \\ Department of Engineering and Design, \\ University of Sussex, \\ Brighton, BN1 9QT \\ United Kingdom
}

\begin{abstract}
A spatial domain optimal trade-off Maximum Average Correlation Height (OT-MACH) filter has been previously developed and shown to have advantages over frequency domain implementations in that it can be made locally adaptive to spatial variations in the input image background clutter and normalised for local intensity changes. In this paper we compare the performance of the spatial domain (SPOT-MACH) filter to the widely applied data driven technique known as the Scale Invariant Feature Transform (SIFT). The SPOT-MACH filter is shown to provide more robust recognition performance than the SIFT technique for demanding images such as scenes in which there are large illumination gradients. The SIFT method depends on reliable local edge-based feature detection over large regions of the image plane which is compromised in some of the demanding images we examined for this work. The disadvantage of the SPOT-MACH filter is its numerically intensive nature since it is template based and is implemented in the spatial domain.
\end{abstract}

Keywords: MACH filter, SIFT filter, correlation filter, invariant pattern recognition

\section{INTRODUCTION}

The major aim of the OT-MACH filter is to find an optimal compromise between good discrimination ability and distortion tolerance in the presence of noise [1]. The MACH filter maximises the relative height of the average correlation peak with respect to the expected distortions. However, the peak height of the MACH filter is unconstrained, making it more difficult to interpret the results of the correlation. The MACH filter is derived by maximising a performance metric called the Average correlation height $(A C H)$. In addition, several other performance measures need to be balanced for better adaptability with different application scenarios. These measures are the Average Correlation Energy ( $A C E$ ), Average Similarity Measure ( $A S M$ ) and Output Noise Variance ( $O N V$ ) [1] , [2].

From equation (1) the resulting transfer function for an OT MACH filter (in the frequency domain) can be seen to be [1]:

$$
h=\frac{m_{x}^{*}}{\alpha C+\beta D_{x}+\gamma S_{x}}
$$

Included in the OT-MACH filter transfer function are: the terms $\alpha, \beta$ and $\gamma$, which are the non-negative weighting parameters; $m_{x}$ which is the average of the training image vector $x_{1}, x_{2}, \ldots, x_{N}$ (in the frequency domain); $C$ which is the diagonal power spectral density matrix of additive input noise and is usually set as the white noise covariance matrix, $C=\sigma^{2} I ; D_{x}$ is the diagonal average power spectral density of the training images [3].

${ }^{1}$ A.A.Gardezi@sussex.ac.uk ; Phone +44 1273873513 ; Fax +44 1273877873 
Previous research has shown that when employing a frequency domain Optimal Trade-off Maximum Average Correlation Height (OT-MACH) filter degradation in performance could be seen when correlations were performed between a uniformly illuminated training dataset and a series of targets having variable illuminations [4].

This degradation in performance is due to the average training image used in the design of the OT-MACH filter. The training image, although beneficial in representing the average behavior of the desired class, nevertheless fails to capture the finer details of the target class. In most cases the average training images tend to look like cluttered images and this may lead to the OT-MACH filter's inability to discriminate the desired class from cluttered backgrounds and hence increase false detection rates. This indicates the extent of variation in the quality of the detection that changes in illumination could cause to the performance of the correlation filter.

In order to further illustrate the degradation in performance of the frequency domain OT-MACH filter in non-uniform illumination, an initial set of experiments have been conducted using a brightly illuminated false target.

In this case the $512 \times 512$ target scene consisted of two targets: a large triangle having pixel intensity of 250; and a $64 \times 64$ square having pixel intensity of 40. The target scene can be seen in Figure 1 given below.

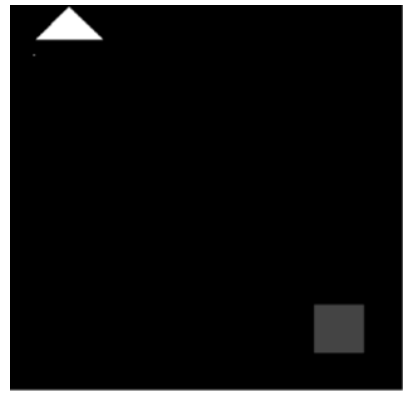

(a)

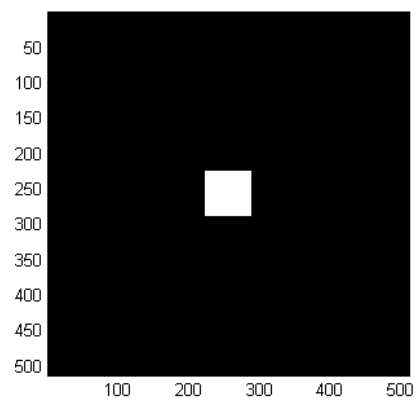

(b)

Figure 1. (a) Target image for frequency domain OT-MACH illumination in-variance test,

(b) Training image for frequency domain OT-MACH

The frequency domain OT-MACH was trained using a 512x512 reference image consisting of a square of $64 \times 64$ pixels having a pixel intensity of 250 in the center of the image. The filter was kept simple with the inclusion of only a single image in the training set, rather than multiple images, in order to check illumination invariance rather than orientation.

When the target image from Figure 1(a) and training image from Figure 1(b) were correlated the output plane shown in Figure 2 below is generated.

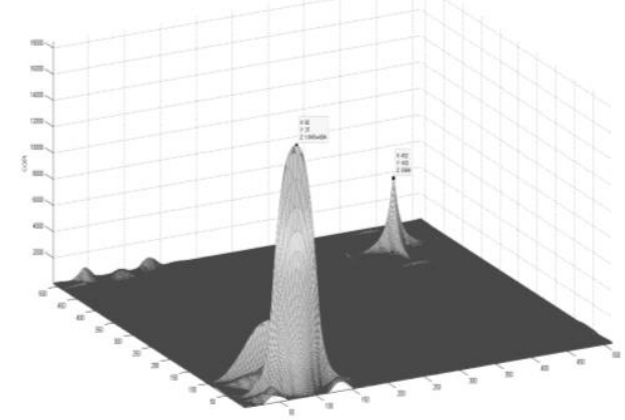

Figure 2. Output plane for OT-MACH in frequency domain

From Figure 2 it can be seen that the Correlation Output Peak Intensity ( COPI ) value for the false target, i.e. the triangle, is $1.847 \times 10^{4}$, and the $C O P I$ for the true target which is the less intensely illuminated square is only $0.5369 \times 10^{4}$. The OT-MACH filter implemented the in frequency domain was tuned with the following parameter settings: $\alpha=0.8$, $\beta=0.9$ and $\gamma=0.7$ which were chosen after an exhaustive set of trials to find the most optimal values. 
From Figure 2 it can be seen that true target has a smaller correlation peak than the false target in the correlation output plane, indicating that the OT-MACH filter has given a false detection of the target object in the presence of non-uniform illumination. Thus in the case where there is a brightly illuminated object present in the scene, the correlation output values will be higher for that region and hence will produce a taller peak which results in a false detection.

In order to overcome this problem a spatial domain approach is proposed for the implementation of the OT-MACH filter which employs local window energy normalisation for the filter to facilitate more tolerance to illumination changes. The proposed filter is named the Spatial Domain OT-MACH (SPOT-MACH) and its design and comparison with other existing feature based techniques is presented in [5] , [6].

\section{Design of the SPOT-MACH filter}

A moving window was used to implement a SPOT-MACH filter which can be locally modified depending upon its position in the input frame. This enables adaptation of the filter dependant on locally variant background clutter conditions and also enables the normalisation of the filter energy levels at each step. Thus the spatial domain SPOT-MACH filter offers an advantage over its frequency domain implementation as shift invariance is not imposed upon it. The only drawback of the spatial domain implementation is the amount of computational resources that are required for its real time implementation. Recently an optical correlator using a scanning holographic memory was proposed by Birch et al for the real time implementation of space variant filters of this type [7].

In order to formulate the transfer function for the SPOT-MACH filter, first the OT-MACH transfer function should be computed in the frequency domain as given by Equation (1). After considering the above mentioned factors, the SPOTMACH filter is then implemented in the spatial domain using a moving kernel to detect an object in a cluttered background plane of size, typically, between $512 \times 512$ and $1024 \times 1024$ pixels.

Normally a white noise covariance matrix is used as additive noise but in this case in order to counter the varying illumination changes, the maximum of the power spectral density of the input image is employed as the constant noise weighting.

The reference image spectrum is inverse Fourier transformed to yield a space domain image as shown in Equation (2):

$$
h=I F F T\left[\frac{m}{\sqrt{\alpha C+\beta D+\gamma S}}\right]
$$

This array is truncated to the target object size which is, typically, less than 256x256 pixels. In addition, care must be taken to store the image as a bi-polar array (rather than an intensity image). The square root operation in the denominator of Equation (2) is required because the filtering operation must also be applied to the input image separately so this is preprocessed with the SPOT-MACH transfer function prior to its spatial domain correlation with the SPOT-MACH kernel function. The use of a moving kernel enables the filter to be adaptive to small areas of the image and hence minimise the impact of varying illumination patterns. Once the images have been passed through a SPOT-transfer function they are correlated using a spatial correlator. In this approach a windowing kernel normally the size of the target object is extracted from the composite image created by the SPOT-MACH and is scanned through the target image pixel by pixel. This enables adaptation of the filter dependant on background illumination variations and also enables the normalisation of the filter energy levels. The kernel can be normalised to remove a non-uniform brightness distribution if this occurs in different regions of the image. The main constraint in this implementation is the dependence on computational ability of the system.

As discussed above, the main advantage of the correlation function is that it can be normalised for amplitude changes using the correlation coefficient in the spatial domain. This is a computationally intensive task and involves calculating a correlation coefficient between two images [8]: 


$$
\varsigma(x, y)=\frac{\sum \sum[f(s, t)-f(s, t)][w(x+s, y+t)-w]}{\left\{\sum \sum[f(s, t)-f(s, t)] \sum \sum[w(x+s, y+t)-w]\right\}^{\prime}}
$$

where $x=0,1,2, \ldots ., M-1$ and $y=0,1,2, \ldots ., N-1$. Also, $\bar{w}$ is the average value of the pixels in the windowed filter function $w$ (which need be evaluated only once) with $\bar{f}$ being the average value of $f$ in the region which contains the moving window. The summations are computed in the regions which are overlapping and have common coordinates for $f$ and $w$. The coefficient of correlation $\varsigma(x, y)$ is scaled in the range of -1 to 1 which is independent to the changes in the amplitude of $f$ and $w$ [8].

\subsection{Operation of SPOT-MACH filter}

When the object is oriented at an unknown angle then $w(x, y)$ in Equation (3) must be rotated so that it aligns with the degree of rotation of $f(x, y)$ which is not feasible if the in-plane rotation is unconstrained. Due to the impracticalities involved in this process, normalized correlation is seldom used in scenarios where unconstrained rotation is present in the target scene [8]. But this limitation can be overcome by the SPOT-MACH transfer function which creates a spatial domain composite image of multiple orientations that can be effectively used as a kernel to perform the normalized correlation with the target scene.

A further point to be noted in the case of the SPOT-MACH filter is the size of the correlation output plane which is smaller as compared to the frequency domain plane. This is due to the fact that the windowing function is used which will effectively return a lesser number of correlation points if zero padding is not used. The correct size of the correlation plane can be established from:

$$
\text { Corr_plane _ } \operatorname{size}(x, y)=(x-\text { window_size, } y \text { - window_size })
$$

Since the correlation output plane size has been compensated, the location of the peak can be made to correspond to the location of the object in the input scene. Thus in order to determine the corrected location of the target object, the following transformation should be applied to the peak coordinates of the SPOT-MACH filter:

$$
\text { Corr_Outputplane }=\left(x-\frac{\text { winsize }}{2}, y-\frac{\text { winsize }}{2}\right)
$$

The use of Equation (5) will now give the exact location of the object in the correlation output plane for the SPOT-MACH filter.

Having established that the SPOT-MACH filter is able to detect similar shaped objects, its capabilities were tested using the same dataset employed in Section 1 for the frequency domain OT-MACH filter. The main aim of this test was to assess the tolerance of the SPOT-MACH filter to the change in illumination when a false target is present in a brightly illuminated area. In order to correctly compare the difference in performance for the frequency domain OT-MACH filter and the SPOTMACH filter, a similar set of reference and target images was thus employed.

In this case, the target image was taken from Figure 1(a) and the reference image from Figure 1(b), i.e. images similar to those used for the OT-MACH frequency domain filter assessment. The SPOT-MACH correlation output plane generated can be seen in Figure 3 below.

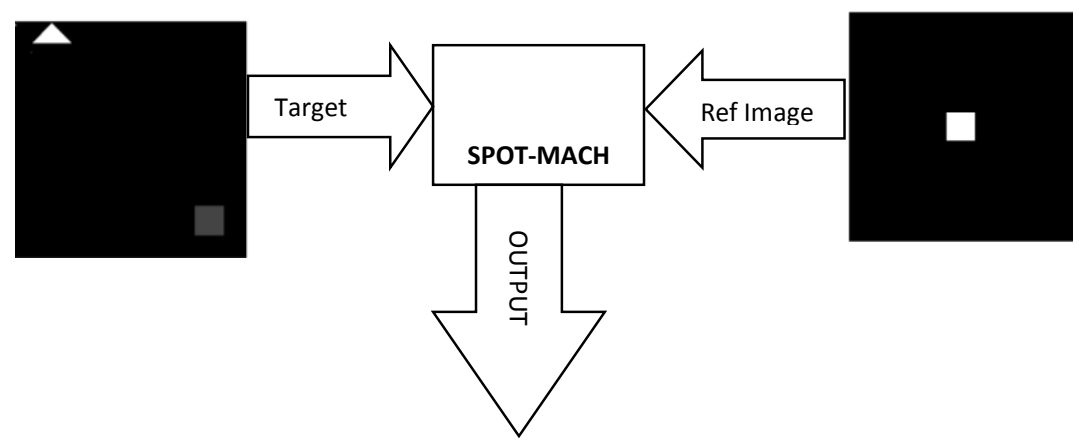




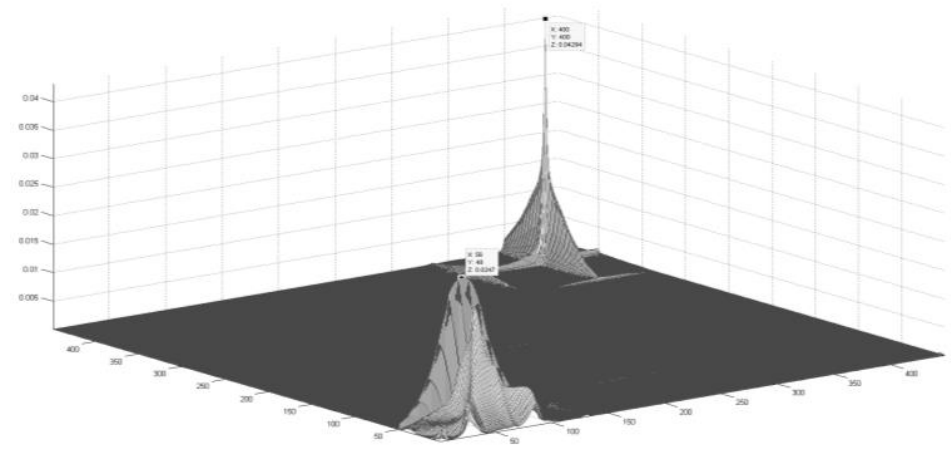

Figure 3. SPOT-MACH correlation output plane for illuminated false target

From Figure 3 it can be seen that the COPI for the false target is 0.024 and for the true target is 0.0429 . This should be compared to the results for the frequency domain OT-MACH, which was unable to detect the true target in the presence of a brightly illuminated false target, whereas the SPOT-MACH is successful.

It can be deduced from this result that even with its improved discrimination capabilities, in this example the frequency domain OT-MACH is unable to distinguish between true and false targets, as shown in Figure 2. The SPOT-MACH achieves this by applying local window energy normalization which effectively suppresses the high energy values of the false target and normalizes with respect to neighbouring pixel intensities.

From Figure 3 it can be seen that the SPOT-MACH is tolerant to changes in illumination and would be effective in situations where the object to be detected might be present in a shaded area. Another major advantage of the SPOT-MACH filter is that the quality of detection is easily verifiable in terms of the highest peak location which corresponds to the detected target coordinates (after being translated using Equation (5)). In contrast, since the frequency domain implementation is unconstrained it is difficult to assess what signifies a good detection and other measures such as $P C E$ and PSR have to be considered to establish criteria for detection.

In order to further analyse the performance of the SPOT-MACH in non-uniformly illuminated environments a series of tests were conducted using visible imagery and the results compared with an existing feature based technique, the Scale Invariant Feature Transform (SIFT) in the following sections of this paper [9] .

\section{Comparison of SPOT-MACH and SIFT using visible imagery}

In order to test the capabilities of SIFT and the SPOT-MACH filter a testing library was created using a car as the target object and varying illumination profiles as background were added. The dataset used consisted of images in which the target was a car oriented at 10 degrees towards the camera from a side-on view.

In total five illumination test cases were prepared which are labeled as follows:

1. Uniform Lighting Test Case

2. Bright Illumination Test Case 
3. Shadow Effect Test Case

4. Spot-Light Test Case

5. Dark Effect Test Case

Each of these test cases have been tested using SIFT, the frequency domain OT-MACH and the SPOT-MACH filter to develop a performance comparison between these approaches.

\subsection{Uniform Lighting Test Case}

In the case of uniform lighting there was a single omni-directional light source having no shadows with a uniform distribution of energy across the plane. There were two scenarios that were created: one with the target object; and another one with the target and an up-scaled false target. The test case created is shown in Figure 4.

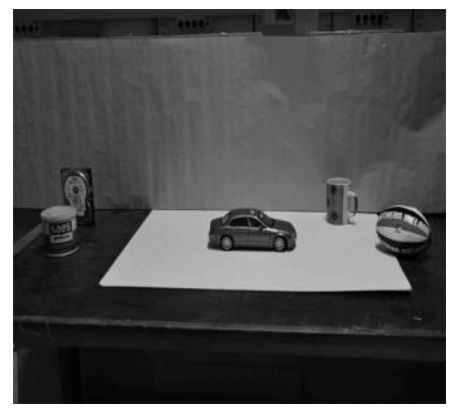

(a)

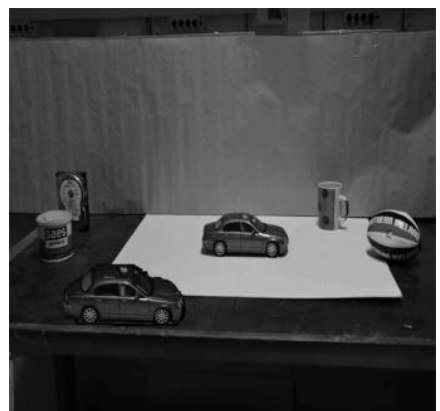

(b)

Figure 4. (a) Uniform Lighting Test Case single target, (b) Uniform Lighting Test Case false target

The test results for Uniform Light test case given by Figure 4 are presented below.

\section{SIFT results for Uniform Lighting Test Case}

The Figure 4 test case was used with the SIFT algorithm and the many successful feature matches indicated in Figure 5 were obtained.
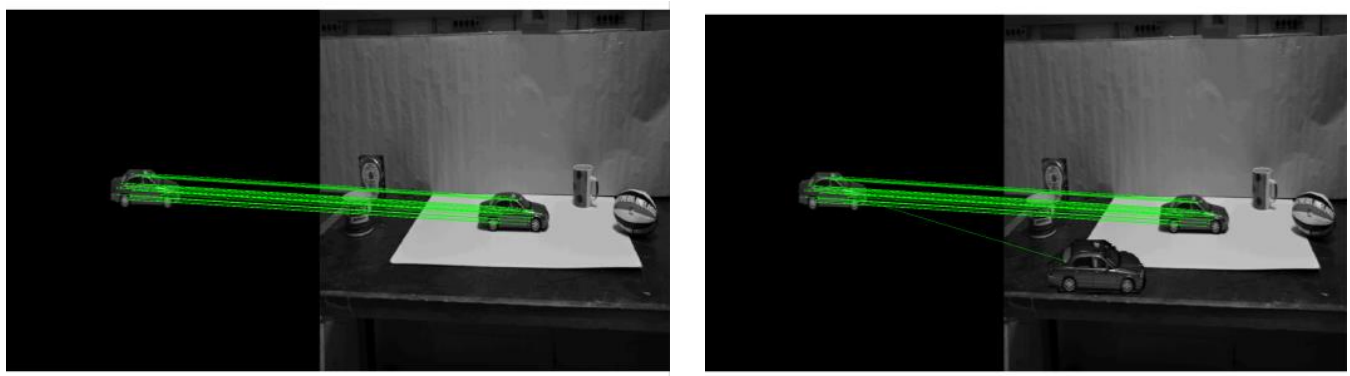

Figure 5. SIFT results for Uniform Lighting Test Case for image 4(a) and 4(b)

\section{Frequency Domain OT-MACH results for Uniform Lighting Test Case}

The frequency domain OT-MACH has been tested using the car image oriented at 10 degrees as the reference image and Figure 4(a) and 4(b) as the target images. The same orientation of the reference and test car image was used to maximize the chances of detection. The clear correlation peaks produced by both images are shown in Figure 6(a) and 6(b).

Figure 6. (a) Output plane for frequency domain OT-MACH for single target, (b) Output plane for frequency domain OT-MACH for two targets

\section{SPOT-MACH results for Uniform Lighting Test Case}

The SPOT-MACH has been tested on the using the car image oriented at 10 degrees as the reference image and Figure 4(a) and 4(b) as the target images producing the clear correlation peaks shown in Figures 7(a) and (b).
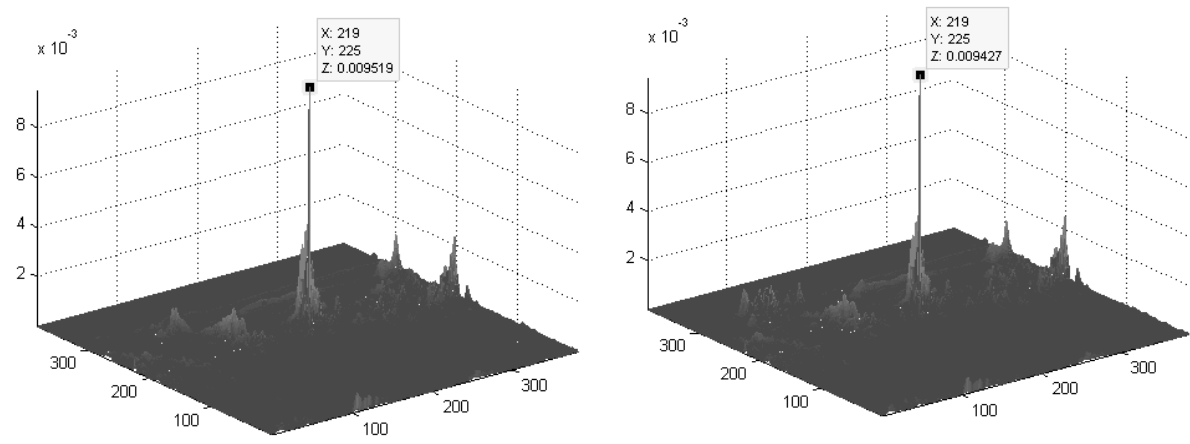

Figure 7. (a) Correlation Output plane for SPOT-MACH for uniform lighting, (b) Correlation Output Plane for SPOT-MACH for uniform lighting with false target.

\subsection{Bright Illumination Test Case}

In the case of Bright Illumination a directional light source has been used which illuminates the target object. In this case there were two scenarios that were created: one with target object; and another one with the target and an up-scaled false target in the non-illuminated area. The test case created is shown in Figure 8.

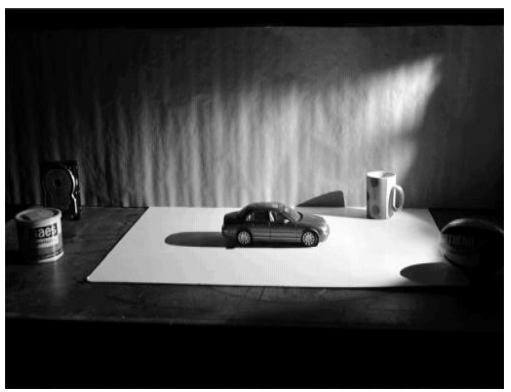

(a)



(b) 
The test results for the bright illumination test case are presented below.

\section{SIFT results for Bright Illumination Test Case}

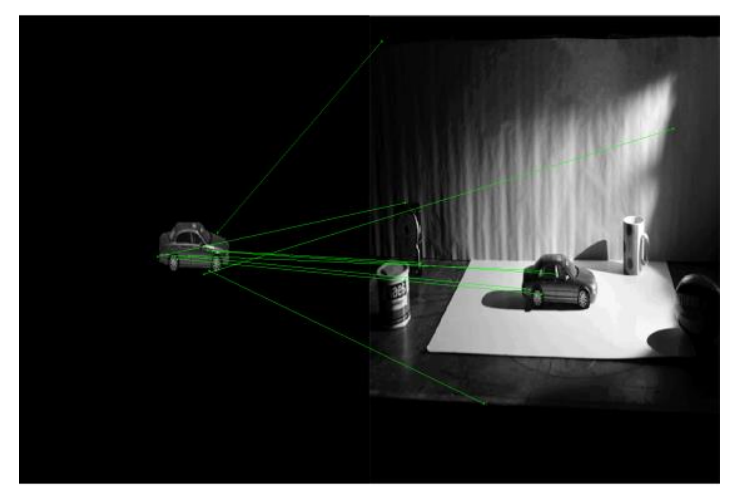

(a)

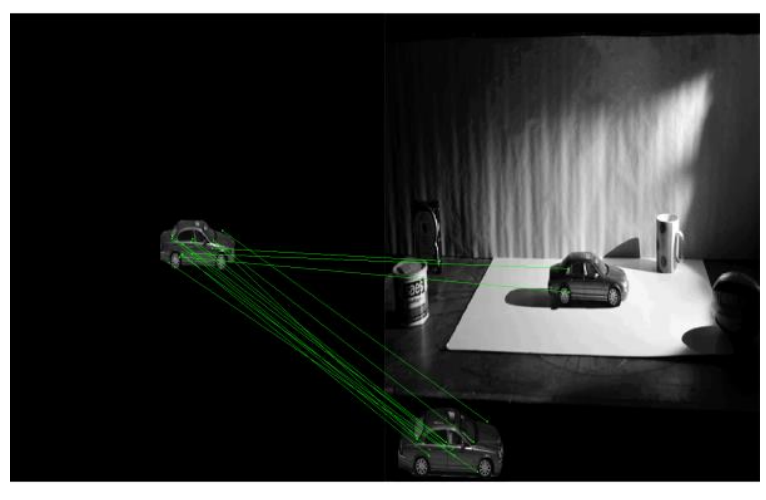

(b)

Figure 9. (a) SIFT results for Bright Illumination Test Case for image 8(a), (b) SIFT results for Bright Illumination Test Case for image 8(b)

The test case was evaluated with the SIFT algorithm and the results shown in Figure 9 (a) and (b) were obtained.

\section{Frequency Domain OT-MACH results for Bright Illumination Test Case}

The frequency domain OT-MACH was tested using the car image oriented at 10 degrees as the reference image and Figure 8(a) and 8(b) as the target image with the results shown in Figure 10.

Figure 10.(a) Output plane for frequency domain OT-MACH for single target in Bright Illumination Test Case, (b) Output plane for frequency domain OT-MACH for two targets in Bright Illumination Test Case

It can be seen from Figure 10(a) and 10(b) that correlation was not successful.

\section{SPOT-MACH results for Bright Illumination Test Case}

The SPOT-MACH has been tested on the using the car image oriented at 10 degrees as the reference image and Figures $8(\mathrm{a})$ and $8(\mathrm{~b})$ as the target images.

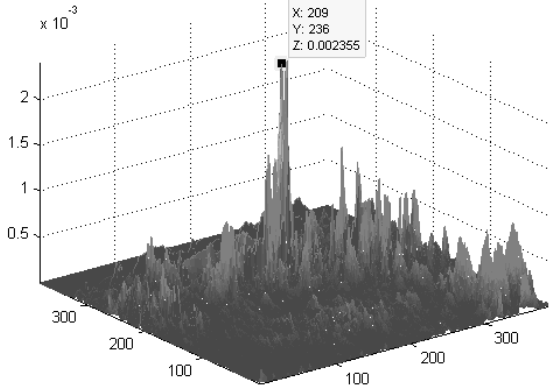

(a)

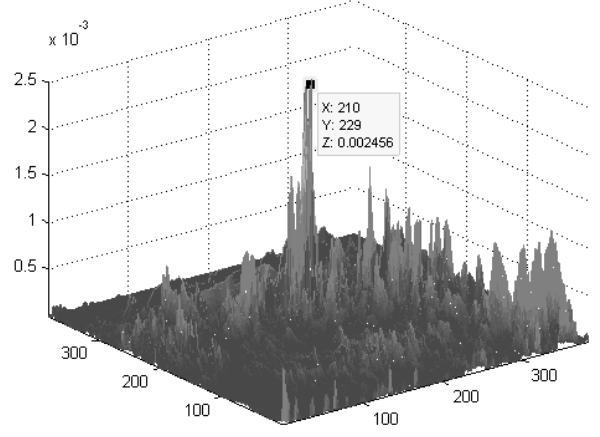

(b)

Figure 11.(a) Correlation Output plane for the SPOT-MACH filter for Bright Illumination, (b) Correlation Output Plane for the SPOT$\mathrm{MACH}$ filter for Bright Illumination with false target

\subsection{Shadow Effect Test Case}

In the testing of a shadow effect an incandescent light source is used along with another similar light source targeted at the car from behind an obstacle to create a shadow effect. In this case there was a part of the car that was under the shadow of the obstacle, with one half of the full image illuminated whilst the other half was under a shadow. The test case created is shown from Figure 12.

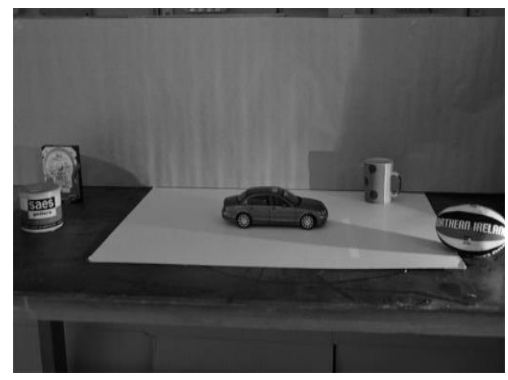

Figure 12. Shadow Effect Test case single target

The results for the Shadow Effect Test case given shown in Figure 12 are presented below. 


\section{SIFT results for Shadow Effect tTest Case}

The test case was evaluated with the SIFT algorithm with the results shown in Figure 13 obtained.

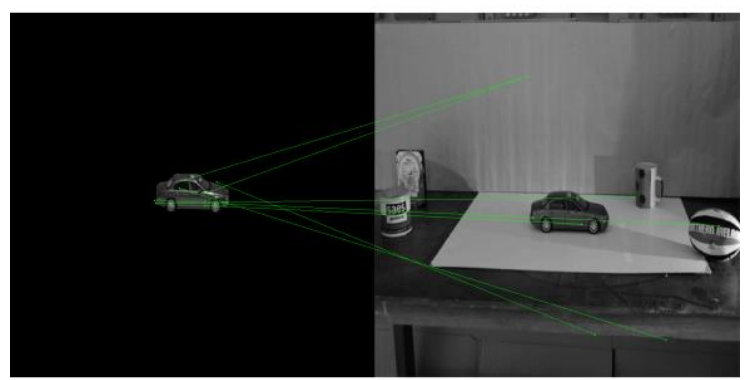

Figure 13. SIFT results for Shadow Effect Test case

From Figure 13 it can be seen that the SIFT fails to give successful matches.

\section{Frequency Domain OT-MACH results for Shadow Effect tTest cCase}

The frequency domain OT-MACH filter was tested using the car image oriented at 10 degrees as the reference image and Figure 12 as the target image.

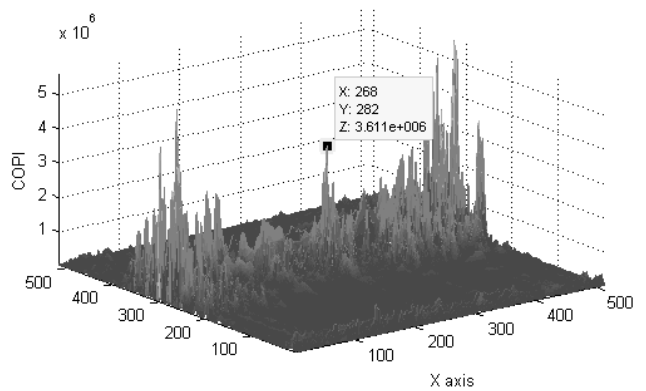

Figure 14. Output plane for frequency domain OT-MACH for Shadow Effect test case

From Figure 14 it can be seen that the OT-MACH fails to detect the target object and generates many false correlation peaks.

\section{SPOT-MACH results Shadow Effect test case}

The SPOT-MACH has been tested with the car image oriented at 10 degrees as the reference image and Figure 12 as the target image.

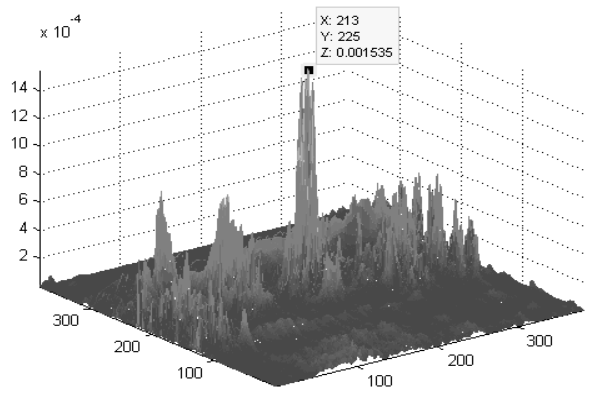


From Figure 15 it can be seen that the SPOT-MACH gives a peak at the location of the target object whereas the previous approaches discussed failed to do so.

\subsection{Spot-Light Effect Test Case}

For a Spot Light Effect test case a dimly lit incandescent light source is used along with another brightly illuminated light source targeted at the front part of the car to produce a spot light effect. The Spot-Light Effect test case so created is shown in Figure 16.

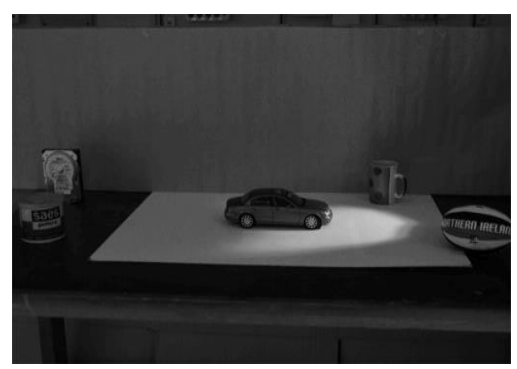

Figure 16. Spot-Light Test Case target image

The results for Spot Light Effect test case shown in Figure 16 are presented below.

\section{SIFT results for Spot Light Effect test case}

The test case was evaluated with the SIFT algorithm and the results shown in Figure 17 obtained.

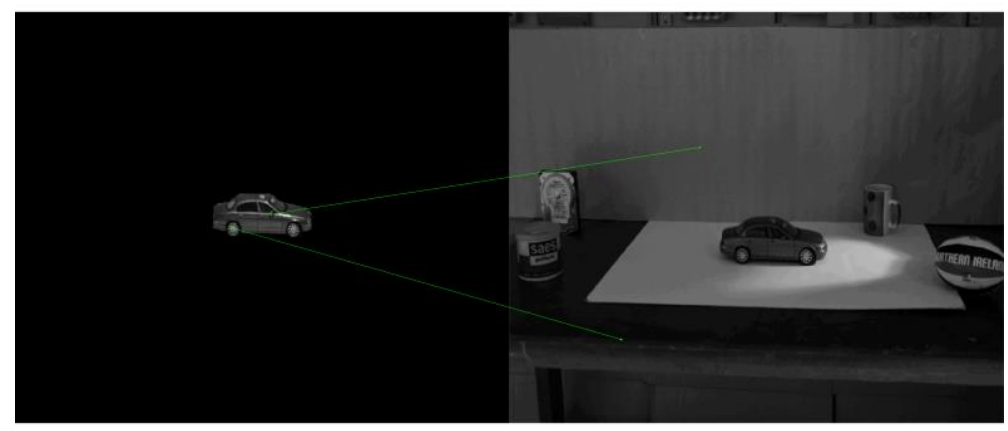

Figure 17. SIFT results for Spot Light Effect Test Case

From Figure 17 it can be seen that the SIFT algorithm fails to produce any reliable matches for this test case.

\section{Frequency domain OT-MACH results for Spot light Test Case}

The frequency domain OT-MACH was tested using the car image oriented at 10 degrees as the reference image and Figure 16 as the target image.




From Figure 18 it can be seen that the OT-MACH produces a detection peak when there is a partly illuminated area present in the scene but also generates a false detection

\section{SPOT-MACH results for Spot Light Effect test case}

The SPOT-MACH was tested the using the car image oriented at 10 degrees as the reference image and Figure 16 as the target image.

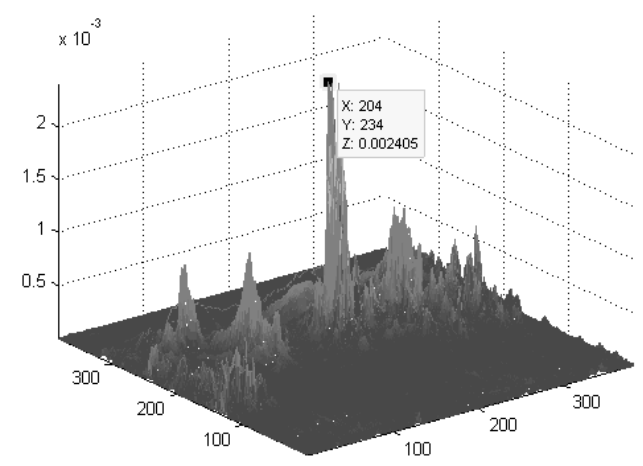

Figure 19. Correlation Output plane for SPOT-MACH for Spot Light Effect Test Case

From Figure 19 it can be seen that the SPOT-MACH produces a detection peak when there is a partly illuminated area present in the scene and effectively suppresses the background clutter to minimize false detections.

\subsection{Dark Effect Test Case}

The Dark Effect test case has been developed to test performance in terms of extreme illumination invariance. In this case the whole of the car is in a dark area and bright light is shown on the wall away from the target to create an area of high energy, generating a scene which can be used as a very demanding test of the performance of the techniques being evaluated.. The Dark Effect test case is shown in Figure 20.

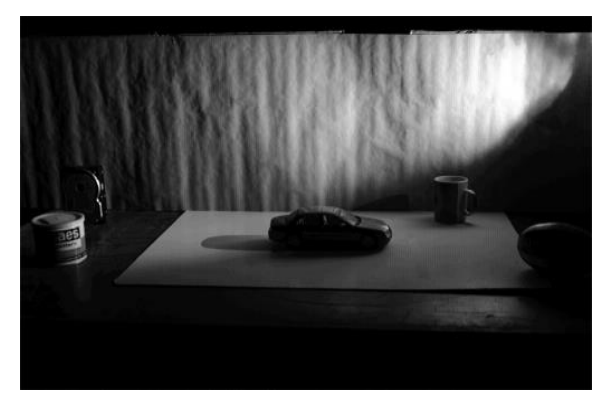

Figure 20. Dark Effect test case target image 
The results for dark effect test case given shown in Figure 20 are presented below.

\section{SIFT results for Dark Effect Test case}

The test case was evaluated with the SIFT algorithm and the results obtained shown in Figure 21 were obtained.

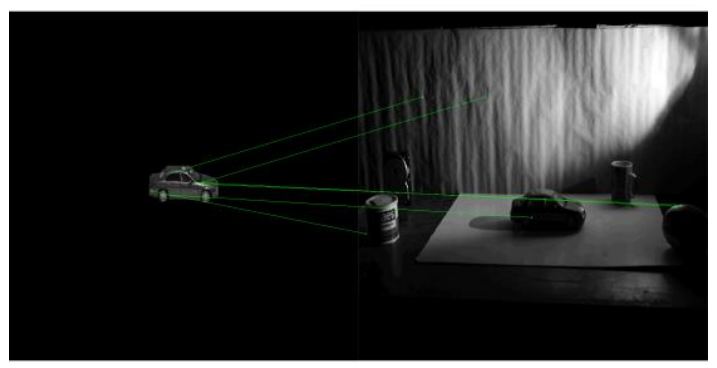

Figure 21. SIFT results for Dark Effect Test Case

From Figure 21 it can be seen that the SIFT produces only a single feature match in the absence of bright light which cannot be classified as a detection as three or more matches are required for reliable identification.

\section{Frequency Domain OT-MACH results}

The frequency domain OT-MACH was tested using the car image oriented at 10 degrees as the reference image and Figure 20 as the target image.

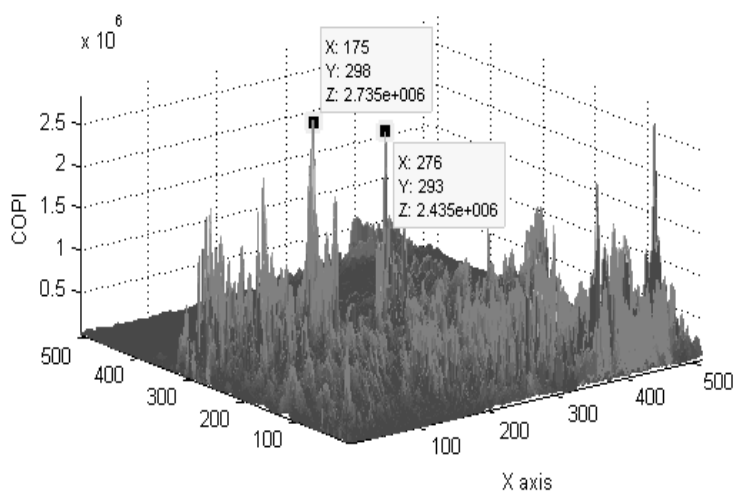

Figure 22. Output plane from frequency domain OT-MACH for the Dark Effect test case

In the Dark Effect test case the OT-MACH filter fails to produce a correlation identification peak due to the low level of the target lighting.

\section{SPOT-MACH filter results for Dark Effect test case}

The SPOT-MACH filter has been tested on the using the car image oriented at 10 degrees as the reference image and Figure 20 as the target image.




Figure 23. Correlation output plane for SPOT-MACH filter for the Dark Effect test case

In the case of the SPOT-MACH filter it can be seen that the location of the target object can be identified by the location of the highest correlation peak, although a number of lower valued false peaks are also generated in the correlation plane.

\section{Summary of Comparative Assessment of Filter Results}

In Section 3 a comparison of the performance of the SPOT-MACH filter with the SIFT feature based technique and the frequency domain implementation of OT-MACH filter was conducted under extremely variable lighting conditions.

Based on the results from these tests, which were specifically developed to assess the performance of these techniques in terms of illumination invariance, a comparison matrix was created which is shown in Table 1 below.

TABLE 1 Comparison Matrix for the OT-MACH, SIFT and SPOT-MACH filters

\begin{tabular}{|llll|}
\hline Test Case & OT-MACH & SIFT & SPOT-MACH \\
\hline Uniform Lighting & PASS & PASS & PASS \\
Bright Illumination & PASS & PASS & PASS \\
Shadow Effect & FAIL & FAIL & PASS \\
Spot Light & PASS & FAIL & PASS \\
Dark Effect & FAIL & FAIL & PASS \\
\hline
\end{tabular}

From Table 1 it can be seen that the SPOT-MACH filter offers the best overall performance in all the scenarios. The SIFT algorithm, although known to be capable of a degree of illumination invariance, was unable to generate matches in situations where a shadow was on the car or a spot light was illuminating a certain region of the car. In the example of the Dark Effect test case, where the target car was completely in a region of very low illumination, only the SPOT-MACH filter was able to detect the exact position of the object in the scene.

This demonstrates that the application of local energy normalisation enables the SPOT-MACH filter to be invariant to changes in illumination. When combined with enhancement techniques, such as inclusion of a space domain nonlinearity[10], the SPOT-MACH filter becomes an at least equally capable technique to feature based techniques.

\section{Conclusion}

One of the main advantages of the SPOT-MACH filter is the ability to allow localised normalisation of the filter which is not possible in the frequency domain implementation of the MACH filter. Results have been presented which indicate a spatial domain implementation of the filter is able to detect, locate and recognise a target object within a non-uniformly scene. Test cases were created, using visual band imagery, containing varying degrees of illumination non-uniformity. These scenes were used for a detailed comparison of the SPOT-MACH filter performance with the SIFT technique and the frequency domain OT-MACH filter to test, in particular, the capability of the filters to detect and recognise a target object despite high degrees of illumination variation. The performance of the different methods was summarised in a table showing a pass-fail matrix from which it is shown that the SPOT-MACH filter is the only filter able to achieve object 
recognition under conditions of extreme illumination variation, outperforming both the frequency domain OT-MACH filter and SIFT algorithm.

\section{References}

1. Mahalonobis, A. , Kumar, B.V.K. , Song, S., Sims, S.R.F., Epperson, J.F., "Unconstrained correlation filters,". Applied Optics 33 , 3751-3759(1994).

2. Goudail, F., Refregier, P., "Optimal Detection of target with random gray levels on spatially disjoint background noise," Opt. Letters 21,495-497(1996).

3. Mahalonobis, A. , Kumar, B.V.K. , "Optimality of the maximum average correlation height filter for detection of target in noise," Optical Engineering 36(1), 2642-2648(1997).

4. Gardezi, A., Birch , P., Young, R. and Chatwin, C. , " Implementation of the Maximum Average Correlation Height $(\mathrm{MACH})$ filter in the spatial domain for object recognition from clutter backgrounds," Proc. SPIE 7696, (2010).

5. Gardezi, A. , Al-Kandri, A. , Birch, P. , Young, R. and Chatwin, C. " A space variant maximum average correlation height (MACH) filter for object recognition in real time thermal images for security applications," Proc. SPIE 7838, (2010).

6. Gardezi, A. , Al-Kandri, A. , Birch, P., Young, R. and Chatwin, C. , " Enhancement of the speed of space-variant correlation filter implementations by using low-pass pre-filtering for kernel placement and applications to real-time security monitoring," Proc. SPIE 8055, (2011).

7. Birch, P., Gardezi, A. ,Mitra,B.K., Young, R. and Chatwin, C., "An optical space domain volume holographic correlator," Proc. SPIE 7340,(2009).

8. Gonzalez,R.C, Woods, R.E., [Digital Image Processing], Prentice-Hall, (2002).

9. Lowe, D.G. " Distinctive image features from scale-invariant keypoints," International Journal of Computer Vision 60(2), 91-110(2004).

10. Jamal-Aldin,L.S., Young, R. and Chatwin, C. , "Application of nonlinearity to wavelet trasnformed images to improve correlation filter performance," Applied Optics 36, 9212-9224 (1997). 